\title{
In Vivo Evaluation of Angiogenic Activity and Its Correlation with Efficacy of Indirect Revascularization Surgery in Pediatric Moyamoya Disease
}

\author{
Yong-il Kim*1-3, Ji Hoon Phi*4, Jin Chul Paeng ${ }^{1}$, Hongyoon Choi ${ }^{1,2}$, Seung-Ki Kim ${ }^{4}$, Yun-Sang Lee ${ }^{1}$, Keon Wook Kang ${ }^{1}$, \\ Ji Yeoun Lee ${ }^{4}$, Jae Min Jeong ${ }^{1,3}$, June-Key Chung ${ }^{1,3}$, Dong Soo Lee ${ }^{1,2}$, and Kyu-Chang Wang ${ }^{4}$ \\ ${ }^{1}$ Department of Nuclear Medicine, Seoul National University College of Medicine, Seoul, Korea; ${ }^{2}$ Department of Molecular Medicine \\ and Biopharmaceutical Sciences, Graduate School of Convergence Science and Technology, Seoul National University, Seoul, Korea; \\ ${ }^{3}$ Cancer Research Institute, Seoul National University College of Medicine, Seoul, Korea; and ${ }^{4}$ Division of Pediatric Neurosurgery, \\ Seoul National University College of Medicine, Seoul, Korea
}

Indirect revascularization is the most widely used treatment to induce angiogenesis in pediatric moyamoya disease (MMD). Molecular imaging methods targeted for angiogenesis have recently been developed. We performed angiogenesis imaging in indirect revascularization surgery for $\mathrm{MMD}$ to evaluate angiogenic activity and its correlation with treatment efficacy. Methods: Twelve patients with pediatric MMD were prospectively enrolled. Encephaloduroarteriosynangiosis surgery was conducted, and ${ }^{68} \mathrm{Ga}-\mathrm{Arg}-$ Gly-Asp (RGD) PET was performed $3.7 \pm 1.0$ mo after surgery. Basal perfusion and stress perfusion $\left(P_{S t r}\right)$ in the middle cerebral artery territory were evaluated by acetazolamide-stress brain perfusion SPECT using statistical probabilistic anatomic mapping, at preoperative, early postoperative, and long-term follow-up states. Angiogenic activity was assessed on the images in terms of maximal uptake ratio, volume of increased uptake, and uptake-volume product. Results: Basal perfusion and $P_{\text {Str }}$ were significantly improved after surgery. Increased angiogenic activity was observed in the revascularized area, mainly around the bony flap. Angiogenic activity gradually decreased with time and significantly correlated with the postoperative time interval $(P=0.0015$ for maximal uptake ratio and 0.0069 for volume of increased uptake). It was estimated to normalize at 6.3 mo after surgery. Uptake-volume product was inversely correlated with $\mathrm{P}_{\mathrm{Str}}$ improvement at the early postoperative state $(r=-0.5960, P=0.0409)$ and also weakly correlated with $\mathrm{P}_{\text {Str }}$ improvement at long-term follow-up $(r=-0.5010, P=0.1165)$. Conclusion: Angiogenesis PET imaging with ${ }^{68} \mathrm{Ga}-\mathrm{RGD}$ was successfully used for the assessment of angiogenic activity in indirect revascularization surgery for MMD, and angiogenic activation measured at approximately 3.7 mo after surgery was inversely correlated with perfusion improvement. The assessment of angiogenic activity using ${ }^{68} \mathrm{Ga}-\mathrm{RGD}$ PET is expected to be effective for evaluating the mechanism or efficacy of revascularization treatment.

Received Jun. 4, 2014; revision accepted Jun. 25, 2014. For correspondence or reprints contact either of the following: Jin Chul Paeng, Department of Nuclear Medicine, Seoul National University College of Medicine, 101 Daehak-ro, Jongno-gu, Seoul, 110-744, Korea.

E-mail: paengic@snu.ac.kr

Kyu-Chang Wang, Division of Pediatric Neurosurgery, Seoul National University College of Medicine, 101 Daehak-ro, Jongno-gu, Seoul, 110-744, Korea.

E-mail: kcwang@snu.ac.kr

${ }^{*}$ Contributed equally to this work.

Published online Jul. 24, 2014.

COPYRIGHT (C) 2014 by the Society of Nuclear Medicine and Molecular Imaging, Inc.
Key Words: moyamoya disease; indirect revascularization; angiogenesis; positron emission tomography

J Nucl Med 2014; 55:1467-1472

DOI: 10.2967/jnumed.114.142430

$\mathbf{M}$ oyamoya disease (MMD) is a progressive occlusive disease of the distal internal carotid artery, and it causes secondary stenosis of the circle of Willis and compensatory abnormal vasculature in the brain (1). The disease presents with cerebral ischemia due to the stenosis and intracerebral hemorrhage from the rupture of abnormal vessels. The disease is relatively common in East Asia including Korea and Japan, and it is the most common pediatric cerebrovascular disease that requires surgical intervention (2). The disease does not respond to any medical treatment, and revascularization surgery is the only effective treatment.

Surgical revascularization for MMD includes direct and indirect methods. In direct revascularization, branches of the internal carotid artery are anastomosed with branches of the external carotid artery. However, in pediatric patients whose vessel is small and fragile, direct revascularization is relatively limited, and indirect revascularization is widely performed (3). In indirect revascularization, vasculature-rich tissues such as the galea, dura, and temporal muscle, are placed on the surface of the brain and angiogenesis of collateral vessels is induced. The indirect method is effective and safe for children (4), although its efficacy is controversial in adults (5). At present, the efficacy of indirect revascularization can be evaluated by cerebral angiography, MR imaging, and brain perfusion SPECT (6), which show final results of collateral vessel formation or cerebral perfusion.

In vivo molecular imaging methods targeted for angiogenesis have recently been developed and used in cancer or ischemic diseases. The tripeptide Arg-Gly-Asp (RGD) has a strong affinity for integrin $\alpha_{v} \beta_{3}$ that is overexpressed on endothelial cells of newly formed vessels (7). RGD peptide has been commonly used as an angiogenesis imaging probe, and several radiolabeled RGD peptides have been developed for SPECT or PET imaging (8-10). We developed a ${ }^{68} \mathrm{Ga}$-labeled RGD peptide $\left({ }^{68} \mathrm{Ga}\right.$-RGD) for PET imaging of angiogenesis (11) and effectively applied it to animal disease models and human diseases (12-14). In indirect revascularization 
of pediatric MMD, the assessment of angiogenic activity by in vivo molecular imaging would be beneficial for investigating the revascularization mechanism and evaluating the efficacy of treatment.

In this study, we evaluated angiogenic activity using in vivo angiogenesis PET imaging in pediatric MMD patients who underwent indirect revascularization surgery. On the angiogenesis PET images, image characteristics, time course of angiogenic activity, and correlation between angiogenic activity and revascularization efficacy were analyzed.

\section{MATERIALS AND METHODS}

\section{Patients and Study Design}

Pediatric patients with newly diagnosed MMD were prospectively enrolled in this study. The following criteria were used for inclusion: MMD confirmed by clinical and radiologic findings; absence of other combined disorders suggesting moyamoya syndrome; absence of previous wide cerebral infarction; and eligibility for standard revascularization surgery and image studies including brain perfusion SPECT, MR imaging, cerebral angiography, and angiogenesis PET. The study design was approved by the Institutional Review Board (IRB) of Seoul National University Hospital (IRB no. H-0908-067-292), and signatures on written informed consent forms were obtained from all parents or legal guardians according to the Declaration of Helsinki.

The overall management methods and surgical outcome of our patients were reported previously (4). All patients underwent preoperative imaging studies including brain MR imaging, cerebral angiography, and brain perfusion SPECT. Revascularization surgery was performed using the unilateral encephaloduroarteriosynangiosis method in which the superficial temporal artery and galeal flap were laid on the surface of the exposed cortex and sutured to the incised edge of the dura mater after dissection of the arachnoid membrane. In some patients, the frontal or occipital area was simultaneously revascularized using the encephalogaleosynangiosis method for the bifrontal area and the encephaloduroarteriosynangiosis method for the occipital area. Angiogenesis PET was performed more than 2 mo after surgery to minimize the effect of postoperative acute inflammation, before the next surgical revascularization for the contralateral side, if any. The patients were closely followed up, and early postoperative brain perfusion SPECT was performed 3 mo after surgery. To evaluate the long-term outcome, brain perfusion SPECT was again performed more than $1 \mathrm{y}$ after surgery.

\section{Image Acquisition}

For angiogenesis PET, ${ }^{68} \mathrm{Ga}$-RGD was prepared in our institution as previously described (11). In brief, a cyclic RGD (cRGDyK) was combined with a derivative of 1,4,7-triazacyclononane-1,4,7-triacetic acid (NOTA). This compound was added to ${ }^{68} \mathrm{Ga}$ eluted from a ${ }^{68} \mathrm{Ge} /{ }^{68} \mathrm{Ga}$ generator, and ${ }^{68} \mathrm{Ga}-\mathrm{RGD}$ was purified from the mixture using highperformance liquid chromatography and alumina sorbent cartridges. ${ }^{68} \mathrm{Ga}$-RGD PET images were acquired using a whole-body PET/CT device equipped with a 40-slice CT scanner (Biograph 40; Siemens Healthcare), which has a PET spatial resolution of $4.2 \mathrm{~mm}$. Patients were injected with ${ }^{68} \mathrm{Ga}$-RGD $(5.5 \mathrm{MBq} / \mathrm{kg})$, and images were acquired $20 \mathrm{~min}$ after the injection. A low-dose CT scan was obtained first for attenuation correction, and PET images were acquired for 30 min using 3-dimensional mode. PET images were reconstructed using an iterative algorithm (ordered-subset expectation maximization; 4 iterations and 8 subsets) on $256 \times 256$ matrices.

Basal/acetazolamide brain perfusion SPECT was performed using a triple-head $\gamma$ camera (Triad XLT 9; Trionix Research Laboratory) equipped with low-energy ultrahigh-resolution fanbeam collimators. Basal SPECT images were acquired 5 min after intravenous injection of ${ }^{99 \mathrm{~m}} \mathrm{Tc}$-hexamethylpropyleneamine oxime $(9.25 \mathrm{MBq} / \mathrm{kg})$. Forty step-and-shoot images were acquired for 20-25 s per step, with intervals of $3^{\circ}$. Ten minutes before the end of the basal SPECT, acetazolamide $(20 \mathrm{mg} / \mathrm{kg})$ was injected intravenously for vasodilation, and ${ }^{99 \mathrm{~m}} \mathrm{Tc}$-hexamethylpropyleneamine oxime $(18.5 \mathrm{MBq} / \mathrm{kg})$ was injected again at the end of the basal SPECT acquisition. Five minutes later, a second acquisition of SPECT was started without position change. Acetazolamide-stress SPECT images were derived by decay-corrected subtraction of the basal images from the second SPECT images. All SPECT images were reconstructed on $128 \times 128$ matrices using a filtered backprojection method with a Butterworth filter.

Brain MR imaging (including MR angiography and perfusion imaging) and cerebral angiography were performed in routine clinical practice before and after the surgery.

\section{Image Analysis}

Angiogenic activity was assessed on PET images in terms of ${ }^{68} \mathrm{Ga}-$ RGD uptake. For quantification, the contralateral temporoparietal brain parenchyma was adopted as a reference region, in which a spheric volume of interest (VOI; $4.04 \mathrm{~cm}^{3}$ ) was drawn to measure the mean and SD of uptake counts. In the revascularized temporoparietal area, an isocontour VOI for increased uptake was drawn using mean +2.5 SDs of the reference region uptake as the margin threshold value. In this VOI, volume and maximal and mean uptake counts were measured. Angiogenic activity was quantified in terms of the maximal uptake ratio $\left(\mathrm{UR}_{\max }\right)$, volume of increased uptake $\left(\mathrm{V}_{\mathrm{IU}}\right)$, and uptake-volume product (UVP), which were defined as follows: $\mathrm{UR}_{\max }=$ (maximal count in the VOI for increased uptake) $/($ mean count in the reference VOI), $\mathrm{V}_{\mathrm{IU}}=$ (size of the VOI for increased uptake in $\mathrm{mm}^{3}$ ), and $\mathrm{UVP}=\left(\mathrm{V}_{\mathrm{IU}}\right) \times($ mean count ratio in the VOI). $\mathrm{UR}_{\max }$ was deemed as the maximal angiogenic activity, $\mathrm{V}_{\mathrm{IU}}$ was deemed as the involved area by angiogenic activation, and UVP was deemed as the overall angiogenic activity. The frontal or occipital area was not included for the analysis even when the area was revascularized during surgery.

The regional brain perfusion in the middle cerebral artery (MCA) territory was assessed on perfusion SPECT. Each SPECT image was spatially normalized to the ethnically matched standard perfusion SPECT template (15) using SPM2 software (University College London) implemented in MATLAB 6.5 (The MathWorks). The probabilistic VOIs for the MCA territory and cerebellum were automatically
FIGURE 1. Improvement in $P_{\mathrm{Bas}}$ and $\mathrm{P}_{\mathrm{Str}}$ after revascularization surgery. $\mathrm{P}_{\mathrm{Bas}}(\mathrm{A})$ and $\mathrm{P}_{\mathrm{Str}}(\mathrm{B})$ were significantly improved from preoperative state (Preop) at early postoperative (Postop) and long-term follow-up (Long-term) states. ${ }^{*}=$ statistically significant changes.

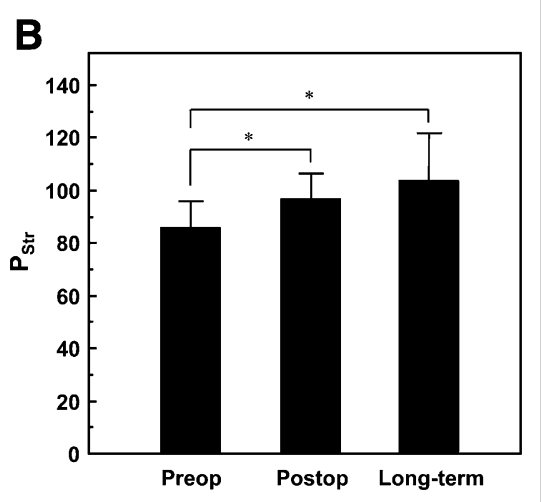




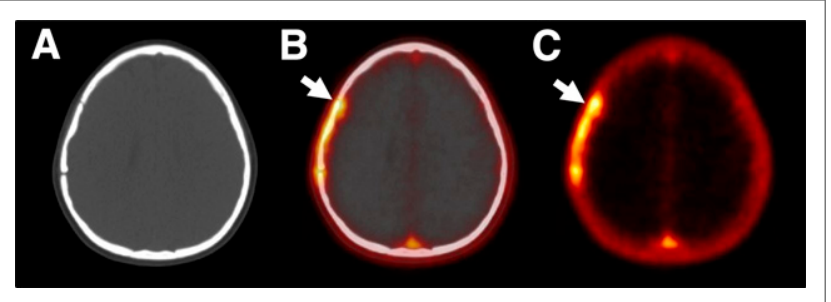

FIGURE 2. ${ }^{88} \mathrm{Ga}-\mathrm{RGD}$ PET/CT images of representative case. On CT (A), fusion (B), and PET (C) images, increased uptake was observed around bony flap, most evidently in craniotomy margin area (arrow). Mild uptake increase was also observed on inner surface of bony flap.

defined using a statistical probabilistic anatomic map (SPAM) (16), and perfusion values of these VOIs were calculated. The perfusion value of the MCA territory was normalized to the mean value of both cerebellar lobes and expressed as a percentage. Basal perfusion $\left(\mathrm{P}_{\mathrm{Bas}}\right)$ and acetazolamide-stress perfusion $\left(\mathrm{P}_{\text {Str }}\right)$ were measured in each MCA territory of basal and acetazolamide-stress SPECT images. $\mathrm{P}_{\text {Str }}$ was deemed as an index for cerebrovascular reserve. The postoperative improvement in $\mathrm{P}_{\mathrm{Bas}}$ and $\mathrm{P}_{\text {Str }}$ was assessed in terms of $\Delta \mathrm{P}_{\mathrm{Bas}}$ and $\Delta \mathrm{P}_{\text {Str }}$, which were defined as [(postoperative measurement) - (preoperative measurement)] in the MCA territory.

\section{Statistical Analysis}

All results were expressed as mean $\pm \mathrm{SD}$. Comparison of 2 group values was performed using the Student $t$ test, and correlation between 2 parameters was evaluated using the Pearson correlation test. $P$ values of less than 0.05 were regarded significant.

Statistical analyses were performed using a commercial software package (MedCalc, version 9.5.0.0; MedCalc Software).

\section{RESULTS}

\section{Patients}

Twelve patients were included in this study ( 9 males and 3 females; mean age $\pm \mathrm{SD}, 7.9 \pm 3.7 \mathrm{y}$; range, $2-15 \mathrm{y}$ ). The patients preoperatively presented with symptoms of transient ischemic attack $(n=11)$, headache $(n=5)$, and seizure $(n=1)$. Four patients had preoperative cerebral infarction in the frontal or occipital area. Revascularization surgery was performed using encephaloduroarteriosynangiosis for the right temporoparietal area and left temporoparietal area in 6 patients each. The frontal area was also revascularized using encephalogaleosynangiosis in 7 patients, and the occipital area was revascularized using encephaloduroar- teriosynangiosis in 2 patients. All patients underwent a second revascularization surgery for the contralateral side $4.0 \pm 1.2 \mathrm{mo}$ (range, 2.4-5.8 mo) after the first surgery. Three patients exhibited postoperative complications of focal infarction or a small amount of subdural hemorrhage (in the right anterior frontal lobe in 2 patients, and in the left high frontal lobe in 1 patient). In the follow-up after revascularization surgery, no patient presented with overt ischemic symptoms related to the operated area.

\section{Perfusion Change}

Preoperative perfusion SPECT was performed $0.6 \pm 0.8$ mo (range, 0.0-2.3 mo) before revascularization surgery, and early postoperative SPECT was performed $3.0 \pm 0.9$ mo (range, 1.9$4.6 \mathrm{mo}$ ) after surgery. Long-term follow-up SPECT was performed $31.7 \pm 7.2$ mo (range, 14.4-40.8 mo) after surgery. In 1 patient, long-term follow-up SPECT was not performed because of intolerance to acetazolamide stress. $\mathrm{P}_{\mathrm{Bas}}$ and $\mathrm{P}_{\mathrm{Str}}$ in the operated MCA territory were $94.3 \pm 9.0$ and $86.0 \pm 9.5$ before revascularization surgery, and they significantly improved to $103.2 \pm 8.8$ and $96.5 \pm$ 9.5 at the first postoperative follow-up $(P=0.0047$ and 0.0064 , respectively). At the long-term follow-up, they were $103.8 \pm 18.2$ and $103.7 \pm 17.3(P=0.0560$ and 0.0020 , respectively, in comparison with preoperative values; Fig. 1).

\section{Angiogenic Activity After Revascularization Surgery}

${ }^{68} \mathrm{Ga}-\mathrm{RGD}$ PET was performed $3.7 \pm 1.0$ mo (range, $2.1-5.3$ mo) after revascularization surgery. On PET images, increased uptake was observed in the operated area, mainly around the bony flap. The uptake was most evident in the craniotomy margin area, and mild uptake increase was also observed on the inner surface (brain side) of the bony flap (Fig. 2).

${ }^{68} \mathrm{Ga}-\mathrm{RGD}$ uptake was time-dependent and gradually decreased with time after revascularization surgery. $\mathrm{UR}_{\max }$ and $\mathrm{V}_{\mathrm{IU}}$ showed significant correlations with the postoperative time interval between revascularization surgery and ${ }^{68}$ Ga-RGD PET $(P=0.0015$ and 0.0069, respectively; Fig. 3). When the linear regression model was applied for extrapolation of $\mathrm{V}_{\mathrm{IU}}$, the uptake was estimated to normalize (reach zero) at 6.3 mo after surgery.

\section{Correlation Between Angiogenic Activity and Revascularization Efficacy}

The interval between ${ }^{68} \mathrm{Ga}$-RGD PET and early postoperative perfusion SPECT was $17 \pm 20 \mathrm{~d}$ (range, $0-54 \mathrm{~d}$ ). On ${ }^{68} \mathrm{Ga}-\mathrm{RGD}$ PET, UVP was significantly correlated with $\Delta \mathrm{P}_{\text {Bas }}$ and $\Delta \mathrm{P}_{\text {Str }}$, with negative correlation coefficients $(r=-0.7189, P=0.0084$, and $r=-0.5960, P=0.0409$, respectively; Figs. $4 \mathrm{~A}$ and $4 \mathrm{~B}$ ). $\mathrm{UR}_{\max }$ and $\mathrm{V}_{\mathrm{IU}}$ showed a tendency of correlation with $\Delta \mathrm{P}_{\mathrm{Bas}}$ and $\Delta \mathrm{P}_{\text {Str }}$, although the correlations were not statistically significant $(P=0.0555$ and 0.0706, respectively; Supplemental Fig. 1 [supplemental materials are available at http://jnm.snmjournals.org]). When the patients were classified by $\Delta \mathrm{P}_{\mathrm{Str}}$ (above and below the median value), UVP was significantly lower in the group with high $\Delta \mathrm{P}_{\mathrm{Str}}$ $(P=0.0390$, Table 1$)$. When the correlation between postoperative time interval and $\Delta \mathrm{P}_{\text {Str }}$ was assessed for consideration of the influence of time, no significant correlation existed between them $(r=0.4294, P=$ 0.1637; Fig. 4C). Additionally, similar

FIGURE 3. Correlation between postoperative time interval and ${ }^{68} \mathrm{Ga-RGD}$ uptake in terms of $U_{R_{\max }}(A)$ and $V_{I U}(B)$. Both indices were significantly correlated with time interval. 


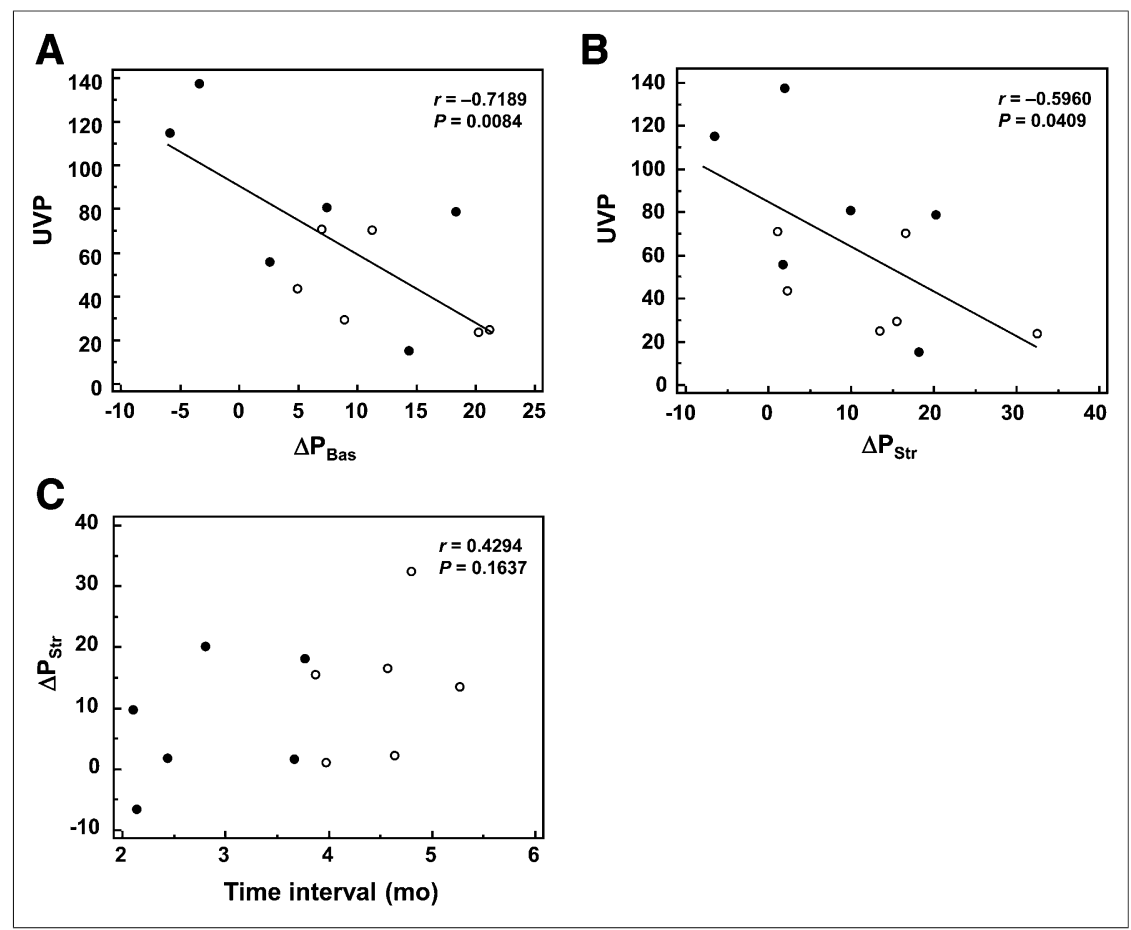

FIGURE 4. Correlation between perfusion improvement and ${ }^{68} \mathrm{Ga}-\mathrm{RGD}$ uptake (A and B), and correlation between postoperative time interval and perfusion improvement (C). UVP was inversely correlated with $\Delta \mathrm{P}_{\mathrm{Bas}}(\mathrm{A})$ and $\Delta \mathrm{P}_{\mathrm{Str}}(\mathrm{B})$, with statistical significances. When patients were classified into groups of relatively shorter $(\bullet)$ and longer $(O)$ postoperative time interval, similar correlation patterns were observed in both groups. $\Delta \mathrm{P}_{\mathrm{Str}}$ had no significant correlation with time interval (C).

correlation patterns were observed between ${ }^{68} \mathrm{Ga}-\mathrm{RGD}$ uptake and $\Delta \mathrm{P}_{\mathrm{Bas}}$ or $\Delta \mathrm{P}_{\mathrm{Str}}$, in both groups when the patients were grouped by the postoperative time interval (above and below the median value; Figs. 4A and 4B). Representative cases with high and low ${ }^{68} \mathrm{Ga}$-RGD uptake are shown in Figure 5 (also in Supplemental Fig. 2).

On comparing ${ }^{68} \mathrm{Ga}-\mathrm{RGD}$ uptake and long-term perfusion change, UVP also showed a tendency of correlation with $\Delta \mathrm{P}_{\mathrm{Bas}}$ and $\Delta \mathrm{P}_{\mathrm{Str}}$ (Fig. 6), although the correlation was not statistically significant $(r=$ $-0.5447, P=0.0832$, and $r=-0.5010, P=0.1165$, respectively).

\section{DISCUSSION}

Angiogenesis and collateral vessel formation is the key mechanism in indirect revascularization surgery for MMD. In the present study, we evaluated angiogenic activity using in vivo angiogenesis PET in revascularization treatment. With the use of ${ }^{68} \mathrm{Ga}$-RGD PET, the pattern of angiogenic activation was visual-

TABLE 1

${ }^{68} \mathrm{Ga}-\mathrm{RGD}$ Uptake According to Perfusion Improvement

\begin{tabular}{lccc}
\hline Image index & High $\Delta \mathrm{P}_{\mathrm{Str}}(n=6)$ & Low $\Delta \mathrm{P}_{\mathrm{Str}}(n=6)$ & $P$ \\
\hline$\Delta \mathrm{P}_{\mathrm{Str}}$ & $19.4 \pm 6.8$ & $1.7 \pm 5.2$ & \\
$\mathrm{UR}_{\max }$ & $2.71 \pm 0.28$ & $3.20 \pm 0.58$ & 0.0897 \\
$\mathrm{~V}_{\mathrm{IU}}$ & $21.9 \pm 15.0$ & $41.2 \pm 15.5$ & 0.0530 \\
UVP & $40.5 \pm 27.1$ & $84.1 \pm 35.9$ & 0.0390
\end{tabular}

Data are mean \pm SD. ized, and angiogenic activity was shown to correlate with the efficacy of revascularization.

In indirect revascularization surgery for MMD, vasculature-rich tissue is placed on the surface of the ischemic brain. Angiogenesis is induced in association with wound-healing and the repair process (17), which is presumably related to the underlying high levels of various growth factors (18). Multiple cranial burr-hole surgery is also performed as an effective adjunct using the same mechanism (19). The efficacy of these treatments is currently assessed by the final outcome of perfusion improvement or new vessel formation using perfusion SPECT, cerebral angiography, or MR imaging (6). However, direct evaluation of angiogenesis would be beneficial for investigating the mechanism of the revascularization process and improving the treatment methods. Recently, radiolabeled RGD peptides were developed for in vivo PET imaging of angiogenesis $(8,9,11)$. Among them, ${ }^{68} \mathrm{Ga}$-labeled RGD has good accessibility because ${ }^{68} \mathrm{Ga}$ can be obtained easily from a generator system, and it has been used for evaluating angiogenic activity in cancer (11), ischemic heart disease (13), and cerebral infarction (12).

In the present study, ${ }^{68} \mathrm{Ga}-\mathrm{RGD}$ PET was performed in indirect revascularization surgery for MMD and exhibited increased activity mainly around the bony flap including adjacent tissue such as galea. The bony flap that covers the operation field is a part of the red marrow-rich skull, and it is in the same vascular territory as that of the revascularized area. Thus, it is speculated that angiogenic activity of the bony flap and adjacent tissue closely correlates with angiogenic activity of the revascularized area. In accordance with this speculation, it has been reported that larger craniotomy is related to better prognosis (20).

It is well known that the uptake of RGD peptides is also enhanced by inflammation (21), because integrin $\alpha_{\mathrm{v}} \beta_{3}$ is expressed not only on endothelial cells of newly formed vessels but also on activated immune cells such as macrophages (22). In the present study, ${ }^{68} \mathrm{Ga}$-RGD uptake possibly correlated with postoperative inflammation, although we performed ${ }^{68} \mathrm{Ga}-\mathrm{RGD}$ PET more than 2 mo after surgery to remove the effect of acute inflammation. The inverse linear correlation between postoperative time interval and ${ }^{68} \mathrm{Ga}$-RGD uptake may have been partially influenced by possible inflammation. However, inflammation is also one of the main processes involved in angiogenesis. In an animal experiment, a fibrous coat enriched with immune cells such as macrophages was the initial pathologic finding in indirect revascularization surgery (17). Also in atherosclerosis, activated monocytes are intimately involved in angiogenesis (23). Thus, increased ${ }^{68} \mathrm{Ga}$-RGD uptake was analyzed without strict discrimination between inflammation and angiogenesis in this study.

With regard to the time course of angiogenic activity, it was estimated to be active until approximately $6 \mathrm{mo}$ from the extrapolation of $\mathrm{V}_{\mathrm{IU}}$. Previous studies have reported that new 


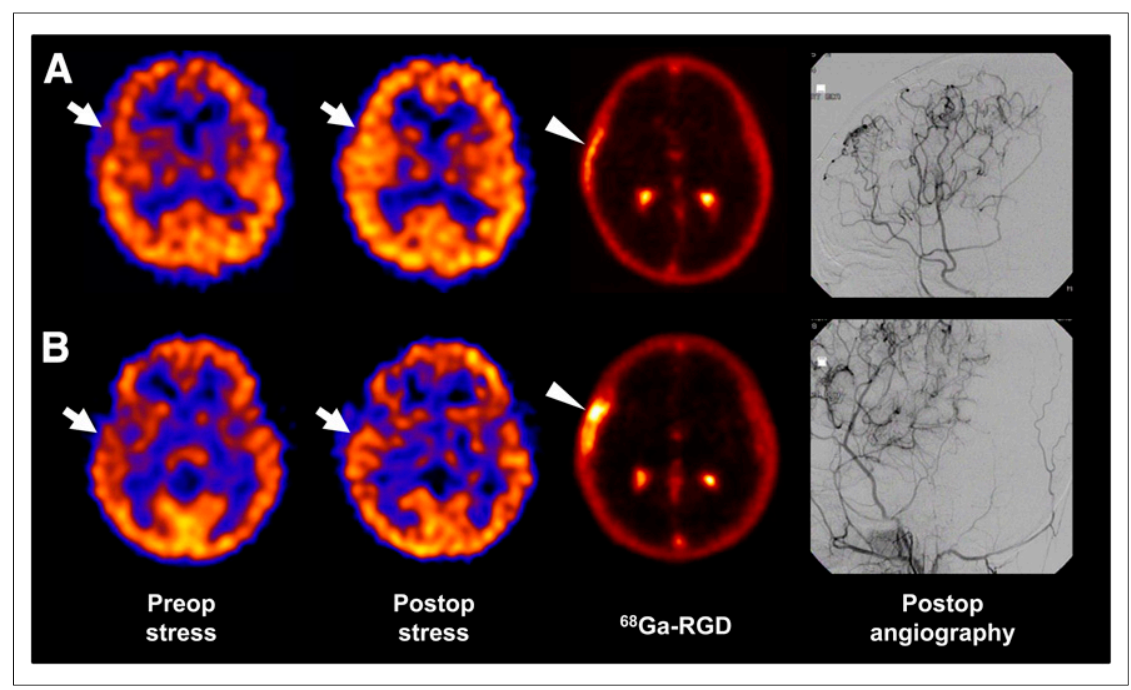

FIGURE 5. Representative cases with high and low ${ }^{68} \mathrm{Ga}-\mathrm{RGD}$ uptake. (A) A 6-y-old boy who underwent encephaloduroarteriosynangiosis surgery for right side. On acetazolamide-stress SPECT, marked improvement of $\mathrm{P}_{\mathrm{Str}}$ in right middle cerebral artery territory (arrow) was exhibited $\left(\Delta \mathrm{P}_{\mathrm{Str}}=32.5\right) .{ }^{68} \mathrm{Ga}-\mathrm{RGD}$ uptake was relatively mild (arrowhead, UVP $\left.=23.8\right)$. (B) Another 6-y-old boy who underwent encephaloduroarteriosynangiosis surgery for right side. Improvement of $P_{S t r}$ in right middle cerebral artery territory (arrow) was relatively mild $\left(\Delta \mathrm{P}_{\mathrm{Str}}=1.9\right)$, whereas ${ }^{68} \mathrm{Ga}$ RGD uptake was marked (arrowhead, UVP $=137.7$ ). In both cases, postoperative angiography for external carotid artery exhibited neoangiogenesis in operated area.

vessels are observed at 3-4 mo after revascularization surgery (24). However, our results suggest that some delayed angiogenesis may occur by 6 mo after surgery. Accordingly, $\mathrm{P}_{\mathrm{Bas}}$ and $\mathrm{P}_{\text {Str }}$ were slightly higher on long-term follow-up SPECT than on immediate postoperative SPECT. The actual time course of angiogenic activity can be assessed by serial follow-up study using noninvasive ${ }^{68} \mathrm{Ga}-\mathrm{RGD} \mathrm{PET}$, and such repetitive evaluation of angiogenic activity would be available within a tolerable range of radiation exposure (25).

One of the intriguing results of our study is that postoperative angiogenic activity was inversely correlated with perfusion improvement. It did not appear to be affected by the time course of angiogenic activity because perfusion improvement did not correlate with the postoperative time interval. In addition, this correlation pattern was observed in both groups when the patients
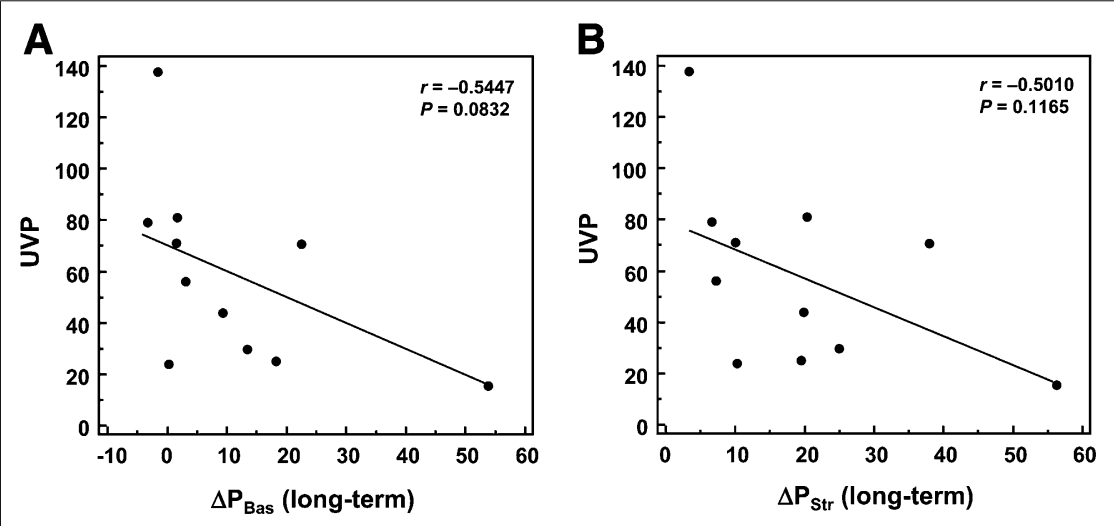

FIGURE 6. Correlation of UVP with $\Delta \mathrm{P}_{\text {Bas }}(\mathrm{A})$ and $\Delta \mathrm{P}_{\mathrm{Str}}(\mathrm{B})$ at long-term follow-up. Perfusion improvement showed tendency of inverse correlation with UVP, although correlation was statistically insignificant. were grouped by the postoperative time interval (Fig. 4). The inverse correlation suggests that the prolonged angiogenic activity may be a result of functionally insufficient revascularization, because ${ }^{68} \mathrm{Ga}-$ RGD PET was performed $3.7 \pm 1.0 \mathrm{mo}$ after surgery. The angiogenic activity was also weakly correlated with the long-term $\mathrm{P}_{\mathrm{Bas}}$ and $\mathrm{P}_{\mathrm{Str}}$ improvement despite statistical insignificance (Fig. 6). Thus, ${ }^{68} \mathrm{Ga}-$ RGD PET could be used as a prognostic factor for the outcome. However, further studies are required for the image pattern and clinical significance of early angiogenic activity after revascularization surgery.

We analyzed volume-related indices of $\mathrm{V}_{\mathrm{IU}}$ and UVP as well as the maximal uptake of $\mathrm{UR}_{\max }$ on ${ }^{68} \mathrm{Ga}-\mathrm{RGD}$ PET. In molecular imaging methods, the overall presence, expression, or activation of the imaging target should be quantified. However, the target region could not be specifically defined and we measured the entire volume that showed uptake above the threshold. Additionally, UVP was calculated to consider both degree and volume of uptake. This approach is widely used in oncologic PET studies to quantify the overall metabolic burden of tumors $(26,27)$. In the present study, UVP that reflects the overall angiogenic activity showed the most significant correlation with $\mathrm{P}_{\mathrm{Bas}}$ and $\mathrm{P}_{\mathrm{Str}}$ improvement among all the indices. Thus, UVP may be regarded as the most sensitive index to measure angiogenic activity on ${ }^{68} \mathrm{Ga}$-RGD PET.

In brain perfusion SPECT, SPAM analysis has been used as an objective and effective method to measure regional perfusion, with an ethnically matched brain template and regional map (16). It may be a limitation of our study that we applied the brain template and probabilistic map obtained from adult brain data to pediatric patients. However, the probabilistic brain map has been applied to pediatric patients without significant errors $(28,29)$. In addition, the VOI of the MCA territory is relatively large; thus, the results would not have been significantly affected by possible minor missegmentation.

There are limitations that should be considered in the present study. First, the case number was small, although we observed statistically significant results. However, multivariate analyses that could show the specific influences of time interval and perfusion improvement on ${ }^{68} \mathrm{Ga}$-RGD uptake were unavailable in this study because of the small case number. Second, the cerebellum was used as a reference tissue for perfusion normalization. It should be considered that the cerebellar perfusion may be affected by crossed cerebellar diaschisis, although the effect is deemed to be minimized with acetazolamide-stress. Additionally, ${ }^{68} \mathrm{Ga}$-RGD PET was performed 1 time at relatively variable time points after the acute phase. These were inevitable because 
clinical conditions and radiation safety should have been considered for pediatric patients, and serial image acquisition is limited. More sophisticated designs are required in further studies in which angiogenesis imaging is used in pediatric MMD patients.

\section{CONCLUSION}

In the present study, ${ }^{68} \mathrm{Ga}$-RGD PET was successfully used for the assessment of angiogenic activity in indirect revascularization surgery for MMD. Angiogenic activity gradually decreases in a time-dependent manner and was estimated to normalize at approximately 6 mo after surgery. Angiogenic activation measured at approximately 3.7 mo after surgery was inversely correlated with perfusion improvement. The assessment of angiogenic activity using ${ }^{68} \mathrm{Ga}-\mathrm{RGD}$ PET is expected to be effective for evaluating the revascularization mechanism or the efficacy of new treatment.

\section{DISCLOSURE}

The costs of publication of this article were defrayed in part by the payment of page charges. Therefore, and solely to indicate this fact, this article is hereby marked "advertisement" in accordance with 18 USC section 1734 . This study was supported by the National Research Foundation of Korea grant funded by the Korean Government (2009-007-6743) and Radiation Technology R\&D program through the National Research Foundation (NRF2012M2A2A7035853). No other potential conflict of interest relevant to this study was reported.

\section{REFERENCES}

1. Fukui M, Kono S, Sueishi K, Ikezaki K. Moyamoya disease. Neuropathology. 2000;20:S61-S64.

2. Kim SK, Wang KC, Kim DG, et al. Clinical feature and outcome of pediatric cerebrovascular disease: a neurosurgical series. Childs Nerv Syst. 2000;16:421-428.

3. Baaj AA, Agazzi S, Sayed ZA, Toledo M, Spetzler RF, Van Loveren H. Surgical management of moyamoya disease. Neurosurg Focus. 2009;26:E7.

4. Kim SK, Cho BK, Phi JH, et al. Pediatric moyamoya disease: an analysis of 410 consecutive cases. Ann Neurol. 2010;68:92-101.

5. Pandey P, Steinberg GK. Neurosurgical advances in the treatment of moyamoya disease. Stroke. 2011;42:3304-3310.

6. Kuroda S, Houkin K. Moyamoya disease: current concepts and future perspectives. Lancet Neurol. 2008;7:1056-1066.

7. Xiong JP, Stehle T, Zhang R, et al. Crystal structure of the extracellular segment of integrin alpha Vbeta3 in complex with an Arg-Gly-Asp ligand. Science. 2002;296:151-155.

8. Decristoforo C, Hernandez Gonzalez I, Carlsen J, et al. ${ }^{68} \mathrm{Ga}$ - and ${ }^{111} \mathrm{In}$-labelled DOTA-RGD peptides for imaging of alpha beta $_{3}$ integrin expression. Eur J Nucl Med Mol Imaging. 2008;35:1507-1515.

9. Li ZB, Wu Z, Chen K, Chin FT, Chen X. Click chemistry for ${ }^{18} \mathrm{~F}$-labeling of RGD peptides and microPET imaging of tumor integrin alpha beta $_{3}$ expression. Bioconjug Chem. 2007;18:1987-1994.

10. Gaertner FC, Kessler H, Wester HJ, Schwaiger M, Beer AJ. Radiolabelled RGD peptides for imaging and therapy. Eur J Nucl Med Mol Imaging. 2012;39(suppl 1): S126-S138.
11. Jeong JM, Hong MK, Chang YS, et al. Preparation of a promising angiogenesis PET imaging agent: ${ }^{68} \mathrm{Ga}$-labeled c(RGDyK)-isothiocyanatobenzyl1,4,7-triazacyclononane-1,4,7-triacetic acid and feasibility studies in mice. J Nucl Med. 2008;49:830-836.

12. Choi H, Phi JH, Paeng JC, et al. Imaging of integrin alpha veta $_{3}$ expression using ${ }^{68} \mathrm{Ga}$-RGD positron emission tomography in pediatric cerebral infarct. Mol Imaging. 2013;12:213-217.

13. Eo JS, Paeng JC, Lee S, et al. Angiogenesis imaging in myocardial infarction using ${ }^{68} \mathrm{Ga}-N O T A-R G D$ PET: characterization and application to therapeutic efficacy monitoring in rats. Coron Artery Dis. 2013;24:303-311.

14. Paeng JC, Lee YS, Lee JS, et al. Feasibility and kinetic characteristics of ${ }^{68} \mathrm{Ga}-$ NOTA-RGD PET for in vivo atherosclerosis imaging. Ann Nucl Med. 2013;27: 847-854.

15. Lee JS, Lee DS, Kim YK, et al. Quantification of brain images using Korean standard templates and structural and cytoarchitectonic probabilistic maps. Korean J Nucl Med. 2004;38:241-252.

16. Kim SJ, Kim IJ, Kim YK, et al. Probabilistic anatomic mapping of cerebral blood flow distribution of the middle cerebral artery. J Nucl Med. 2008;49: 39-43.

17. Nakamura M, Imai H, Konno K, et al. Experimental investigation of encephalomyosynangiosis using gyrencephalic brain of the miniature pig: histopathological evaluation of dynamic reconstruction of vessels for functional anastomosis. Laboratory investigation. J Neurosurg Pediatr. 2009;3:488-495.

18. Weinberg DG, Arnaout OM, Rahme RJ, Aoun SG, Batjer HH, Bendok BR. Moyamoya disease: a review of histopathology, biochemistry, and genetics. Neurosurg Focus. 2011;30:E20.

19. McLaughlin N, Martin NA. Effectiveness of burr holes for indirect revascularization in patients with moyamoya disease: a review of the literature. World Neurosurg. 2014;81:91-98.

20. Kuroda S, Houkin K, Ishikawa T, et al. Determinants of intellectual outcome after surgical revascularization in pediatric moyamoya disease: a multivariate analysis. Childs Nerv Syst. 2004;20:302-308.

21. Cao Q, Cai W, Li ZB, et al. PET imaging of acute and chronic inflammation in living mice. Eur J Nucl Med Mol Imaging. 2007;34:1832-1842.

22. Waldeck J, Hager F, Holtke C, et al. Fluorescence reflectance imaging of macrophage-rich atherosclerotic plaques using an $\alpha_{\mathrm{v}} \beta_{3}$ integrin-targeted fluorochrome. J Nucl Med. 2008;49:1845-1851.

23. Jaipersad AS, Lip GY, Silverman S, Shantsila E. The role of monocytes in angiogenesis and atherosclerosis. J Am Coll Cardiol. 2014;63:1-11.

24. Houkin K, Nakayama N, Kuroda S, Ishikawa T, Nonaka T. How does angiogenesis develop in pediatric moyamoya disease after surgery? A prospective study with MR angiography. Childs Nerv Syst. 2004;20:734-741.

25. Kim JH, Lee JS, Kang KW, et al. Whole-body distribution and radiation dosimetry of ${ }^{68} \mathrm{Ga}-\mathrm{NOTA}-\mathrm{RGD}$, a positron emission tomography agent for angiogenesis imaging. Cancer Biother Radiopharm. 2012;27:65-71.

26. Choi ES, Ha SG, Kim HS, Ha JH, Paeng JC, Han I. Total lesion glycolysis by ${ }^{18} \mathrm{~F}$-FDG PET/CT is a reliable predictor of prognosis in soft-tissue sarcoma. Eur J Nucl Med Mol Imaging. 2013;40:1836-1842.

27. Kim TM, Paeng JC, Chun IK, et al. Total lesion glycolysis in positron emission tomography is a better predictor of outcome than the International Prognostic Index for patients with diffuse large B cell lymphoma. Cancer. 2013;119:11951202.

28. Lee HY, Lee JS, Kim SK, et al. Assessment of cerebral hemodynamic changes in pediatric patients with moyamoya disease using probabilistic maps on analysis of basal/acetazolamide stress brainperfusion SPECT. Nucl Med Mol Imaging. 2008;42:192-200.

29. Song YS, Oh SW, Kim YK, Kim SK, Wang KC, Lee DS. Hemodynamic improvement of anterior cerebral artery territory perfusion induced by bifrontal encephalo(periosteal) synangiosis in pediatric patients with moyamoya disease: a study with brain perfusion SPECT. Ann Nucl Med. 2012;26:47-57. 